\title{
From in vitro-in vivo relationship (IVIVR) towards in vitro-in vivo extrapolation (IVIVE): A case study of pulmonary delivery systems
}

\author{
Paweł Konrad Tuszyński ${ }^{1}{ }^{*}$, Sebastian Polak ${ }^{2,3}$, Renata Jachowicz $^{1}$, Aleksander Mendyk ${ }^{1}$ \\ ${ }^{1}$ Department of Pharmaceutical Technology and Biopharmaceutics, Faculty of Pharmacy, Jagiellonian University Medical College, Kraków, Poland \\ ${ }^{2}$ Faculty of Pharmacy, Jagiellonian University Medical College, Kraków, Poland \\ ${ }^{3}$ Simcyp Ltd. (a Certara Company), Blades Enterprise Centre, John Street, Sheffield, South Yorkshire, UK
}

\section{ABSTRACT}

Based on the results from the literature, a direct relationship between dissolution profiles and in vivo markers of low molecular weight heparin (LMWH) concentration was discovered by application of the RIVIVR tool. Previously, LMWH-loaded microspheres were administered intratracheally to rats and anti-factor Xa activity was measured as a surrogate of $\mathrm{LMWH}$ actual concentration in blood. A non-compendial dissolution method was employed to produce in vitro dissolution profiles. Four microsphere formulations were taken into consideration, and RIVIVR was employed to produce direct relationships between dissolution profiles and anti-Xa factor activity of heparin. Four experiments were performed, and each time one formulation was excluded for external validation. Internal and external validation errors were computed as prediction errors (PEs) (\%). In the case of all four formulations, all of PEs were below $10 \%$, both in internal and external validation procedures. The results indicate good quality of the models and flexibility of the RIVIVR tool, whereas IVIVR (in vitro-in vivo relationship) is built without intravenous administration data. Moreover, as anti-Xa factor activity is no direct measurement of heparin concentration, the results confirm usability of RIVIVR for nonclassical pharmacokinetic data for the in vivo part of IVIVR.

KEYWORDS: In vitro-in vivo correlation (IVIVC); in vitro-in vivo relationship (IVIVR); pulmonary delivery system; dissolution

\section{BACKGROUND}

D ulmonary drug delivery is an effective way of drug administration during the treatment of the respiratory diseases as well as systematic diseases. When a drug is acting as a systematic agent, one can treat such approach as a noninvasive 'needle-free' delivery system. Pulmonary delivery is suitable for a variety of small substances and is especially useful when drugs with high first-pass metabolism are considered. An environment independent of diet-driven modifications with low enzymatic activity, and free of hepatic or intestinal firstpass effect, assures reproducible absorption kinetics. Additionally, low lung clearance allows for applying sustained-release formulations with prolonged release of a drug $(1,2)$.

A reproducible and reliable method of modeling the drug bioperformance is needed if pulmonary delivery of the systemic agent is to be considered. Moreover, in this environment, non-linear relationships should be expected if pharmacokinetic (PK) data are being predicted. RIVIVR, a tool described previously, was applied to model the data obtained from literature with low molecular weight heparin (LMWH) considered as a model systemic agent $(3,4)$. RIVIVR, as an empirical modeling tool, can handle such data without going into mechanistic details of the process if enough data are provided from in vivo and in vitro studies.

Generally, IVIVR (in vitro-in vivo relationship) is an effort to relate in vitro dissolution data to in vivo PK data, and IVIVE (in vitro-in vivo extrapolation) is a broader term that aims to predict phenomena on full living organisms based on experimental results or observations. In this case, an actual pharmacodynamic (PD) parameter was modeled because anti-Xa activity, which describes the anticoagulant properties of heparin, was measured. Thus, in our approach, a direct relationship between dissolution profiles and in vivo markers of $\mathrm{LMWH}$ concentration was investigated, as a surrogate of actual LMWH concentration in blood.

\section{METHODOLOGY}

\section{Data}

Data were obtained from studying the feasibility of large porous particles as long-acting carriers for pulmonary delivery of LMWH. Poly(DL-lactide-co-glycolide) (PLGA) 
microspheres were loaded with LMWH of $61 / \mathrm{mg}$ antifactor Xa activity. Particles were obtained with an evaporation method using dichloromethane as an evaporating solvent. Obtained microspheres were washed with distilled water and lyophilized (freeze-dried) to obtain free-flowing powder (4).

\section{Dissolution study}

A non-compendial dissolution method was employed to produce in vitro dissolution profiles. The amount of $50 \mathrm{mg}$ of freeze-dried microspheres was suspended in $10 \mathrm{~mL}$ phosphate-buffered saline with the addition of $0.1 \%$ polysorbate 80 . Magnetic stirring of $200 \mathrm{rpm}$ was employed. Subsequently, samples were taken and were centrifuged. Supernatant was removed and assayed for heparin content with a colorimetric method.

In vivo study

Male Sprague-Dawley rats (Charles River Laboratories, Charlotte, NC, USA) were used to measure in vivo phenomena. Measurements were taken indirectly, through plasma anti-factor Xa activity.

The animal plasma was added to a known amount of excess factor $X a$. If heparin is present in the plasma, it binds to antithrombin and forms a complex with factor Xa. The amount of residual factor $\mathrm{Xa}$ is inversely proportional to the amount of heparin. The residual factor $\mathrm{Xa}$ is detected by adding a substrate that mimics the natural substrate of factor Xa. This is cleaved by the residual factor Xa, releasing a colored compound (chromophore) that can be detected by a spectrophotometer, resulting in the antifactor Xa activity profile.

The study microspheres were administered intratracheally $(50 \mathrm{U} / \mathrm{kg}$ ) by using a specially designed dry-powder insufflator (Penn Century, Philadelphia, PA, USA) for aerosol inhalation in small animals. Plasma obtained from untreated rats was used as a negative control to account for the effect of rat endogenous anti-factor Xa that otherwise could give a false positive increase in antifactor Xa activity.

As stated by the authors of the original study, correlation of the in vitro release with the release of API in the lungs can be challenging since the medium used for in vitro tests differed significantly from the actual lung fluid (5). However, with the empirical approach presented here, if the model is validated, it can be assumed that predictability can be achieved on the satisfactory level.

Four formulations were considered, named PM-2, PMSP-2, PM-SA-2, and PM-PEI-2 (4). The mean diameters of the particles were $6.77,6.65,9.85$, and $11.01 \mu \mathrm{m}$, respectively. Each one of them consisted of $50 \mathrm{mg}$ of LMWH encapsulated in $250 \mathrm{mg}$ of PLGA microspheres, which differed in terms of the internal aqueous phase (IAP) to organic phase (OP) to external aqueous phase (EAP) ratio used to prepare the microspheres, which was 0.25:5:25 for PM-2 and 0.5:5:25 for other three formulations, respectively. PM-SP-2, PM-SA-2, and PMPEI-2 contained core-modifying agents in the IAP: sorbitan monostearate to stearylamine to polyethyleneimine, respectively.

\section{Modeling procedure}

Similarly to the case of IVIV correlations, an assumption was made that a direct relationship between in vitro and in vivo data can be established (5).

RIVIVR is a new tool for a direct, convolution-based, correlation of dissolution profiles with their PK counterparts. It is based on the optimization approach where optimized parameters are points of numerically developed intravenous (IV) time-concentration curve (in silico IV profile) used for convolution of the PK profile representing oral administration of the particular formulation. Once optimized on two or three available formulations, the in silico IV profile is used for convolutionbased prediction of a new formulation PK profile based on the new dissolution profile, thus accounting for the external validation.

The algorithm of RIVIVR execution is represented by following pseudo-code:

1: Gather in vitro and in vivo data (at least 2 formulations with different release rates)

2: Step $=1$

3: IF step $=1$, THEN

initialize artificial IVIV profile (impulse)

\section{ELSE}

update artificial IVIV profile from p. 5

4: Step = step +1

5: Modify artificial IVIV profile

6: Convolve artificial IVIV profile with in vitro profile

7: Compare convolved PK profile with its corresponding observed counterpart

8: Compute prediction error upon comparison

9: IF prediction error > stop criterion, THEN

go back to p. 3

ELSE

exit. 
Four experiments were performed, and each time one formulation was excluded for external validation. Internal and external validation errors were computed as prediction errors (PEs) (\%).

\section{RESULTS AND DISCUSSION}

In the case of all studied formulations, PEs were below $10 \%$, both in internal and external validation procedure. This is a confirmation of good quality of the models. Table 1 shows external PEs.

Table 1. Prediction Errors (PE) for the External Validation Procedure

\begin{tabular}{|c|c|c|c|}
\hline Formulation & $\mathbf{R}^{2}$ & PE_Cmax (\%) & PE_AUC (\%) \\
\hline PM-2 & 0.795 & 8.22 & 0.14 \\
\hline PM-SP-2 & 0.841 & 3.92 & 2.07 \\
\hline PM-SA-2 & 0.941 & 5.37 & 1.84 \\
\hline PM-PEI-2 & 0.995 & 1.52 & 0.95 \\
\hline
\end{tabular}

Profiles given as an input differed between each other, which guarantees good generalization abilities of the prediction model. Predicted profiles of Xa activity are presented in Figure 1.

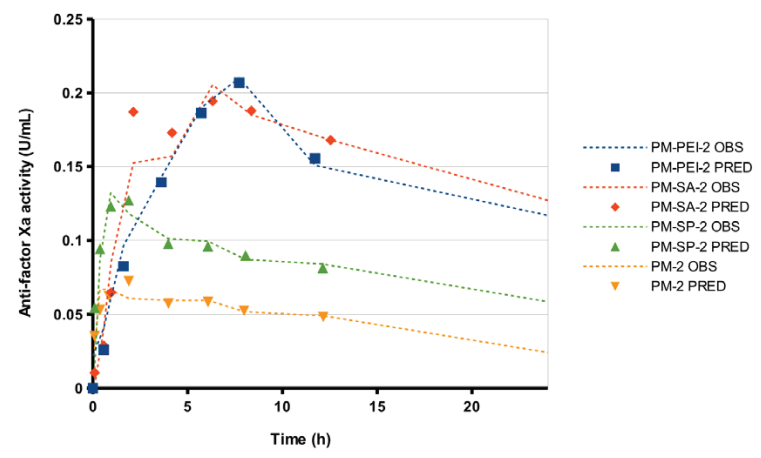

Figure 1. Predicted (PRED) anti-factor Xa activity profiles and observed (OBS) values.

Figure 2 shows the predicted versus observed plots with R-squared for each prediction validation and the overall, which was 0.948 , again demonstrating good quality of predictions.

On Figure 3, the summary of steps mentioned above are depicted. In each experiment, only three formulations were used to calculate and optimize artificial impulse IV profile, upon which the Xa activity was predicted. Presented calculated impulse profiles (Figure 4) have no physical meaning, being only a scaling factor for the numerical convolution procedure. As RIVIVR is purely empirical tool, loss of mechanistic understanding of the "impulse" curve is a price for good predictability of the model described above.

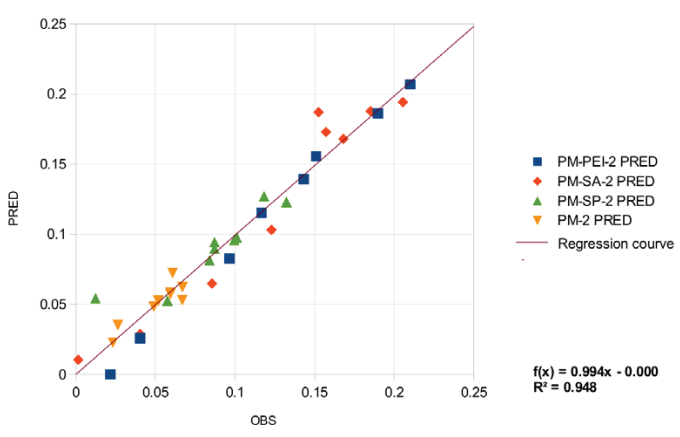

Figure 2. Predicted (PRED) versus observed (OBS) scatter-plot for all formulations. Solid line presents linear model and R2 of all formulations.

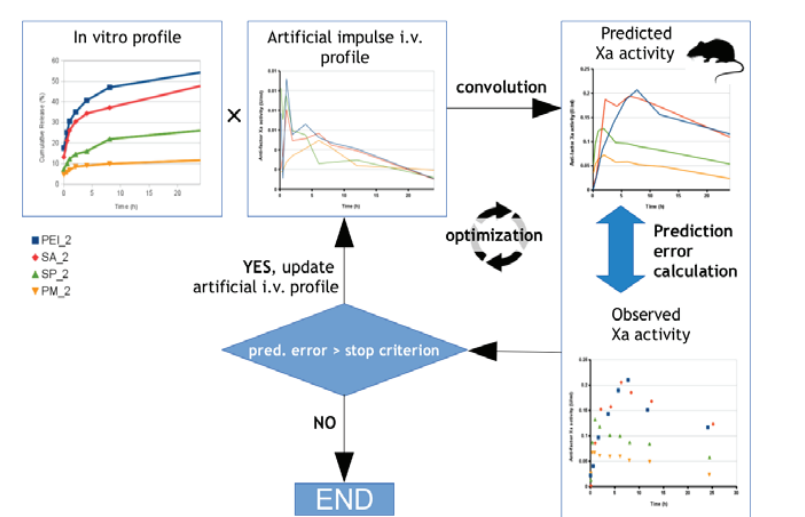

Figure 3. Optimization procedure explained on the given dataset.

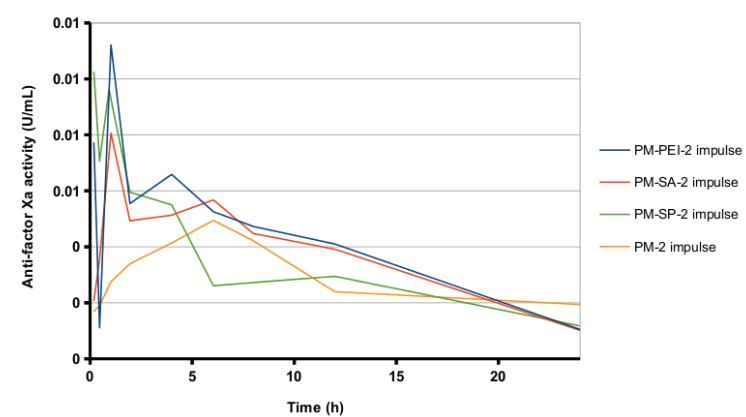

Figure 4. Generated impulse profiles.

\section{CONCLUSIONS}

We have demonstrated the usefulness of the RVIVR tool in the modeling of the complex relationship between in vitro and in vivo data. Results indicate good quality of the models and flexibility of the RIVIVR tool, whereas IVIVR is built without intravenous administration data. Moreover, as anti-Xa factor activity is no direct measurement of heparin concentration, the above results confirm usability of RIVIVR for nonclassical PK data for the in vivo part of IVIVR. 
The results of the current study correspond well to the recent advancements in model-based drug development $(6,7)$. In general, the in vitro-in vivo extrapolation relates to either PK or PD effects scaling from in vitromeasured ADME (absorption, distribution, metabolism and excretion) parameters or compound activity data for $\mathrm{PK}$ and PD, respectively, to the in vivo situation $(8,9)$. The novel approach presented here offers new possibilities in the situation when limited data are available.

It is noteworthy that not only PK but also pharmacodynamic effects were predicted in this case, solely based on microspheres dissolution data. Therefore, a simple in vitro/PD model was established.

\section{CONFLICT OF INTERESTS}

The authors declare no conflict of interest.

\section{REFERENCES}

1. Labiris, N. R.; Dolovich, M. B. Pulmonary drug delivery. Part I: Physiological factors affecting therapeutic effectiveness of aerosolized medications. Br. J. Clin. Pharmacol. 2003, 56 (6), 588-599. DOI: 10.1046/j.1365-2125.2003.01892.x.

2. Labiris, N. R.; Dolovich, M. B. Pulmonary drug delivery. Part II: The role of inhalant delivery devices and drug formulations in therapeutic effectiveness of aerosolized medications. $\mathrm{Br}$. J. Clin. Pharmacol. 2003, 56 (6), 600-612. DOI: 10.1046/j.13652125.2003.01893.x.

3. Mendyk, A. R scripts for multivariate analysis. RIVIVR software.
Sourceforge website. Slashdot Media. http://sourceforge.net/ projects/rscriptsmultivariate/files/RIVIVR (updated April 16, 2014; accessed April 16, 2015).

4. Rawat, A.; Majumder, Q. H.; Ahsan, F. Inhalable large porous microspheres of low molecular weight heparin: In vitro and in vivo evaluation. J. Control. Release 2008, 128 (3), 224-232. DOI: 10.1016/j.jconrel.2008.03.013.

5. Mendyk, A.; Tuszyński, P. K.; Khalid, M. H.; Jachowicz, R.; Polak, S. How-to: Empirical IVIVR without intravenous data. Dissolution Technol. 2015, 22 (2), 12-18. DOI: 10.14227/DT220215P12.

6. Zhang, L.; Pfister, M.; Meibohm, B. Concepts and challenges in quantitative pharmacology and model-based drug development. AAPS J. 2008, 10 (4), 552-559. DOI: 10.1208/s12248-008-90623.

7. Zhang, L.; Sinha, V.; Forgue, S. T.; Callies, S.; Ni, L.; Peck, R.; Allerheiligen, S. R. B. Model-based drug development: The road to quantitative pharmacology. J. Pharmacokinet. Pharmacodyn. 2006, 33 (3), 369-393. DOI: 10.1007/s10928-006-9010-8.

8. Burt, H. J.; Neuhoff, S.; Almond, L.; Gaohua, L.; Harwood, M.; Jamei, M.; Rostami-Hodjegan, A.; Tucker, G. T.; RowlandYeo, K. Metformin and cimetidine: Physiologically based pharmacokinetic modelling to investigate transporter mediated drug-drug interactions. Eur. J. Pharm. Sci. 2016, 88, 70-82. DOI: 10.1016/j.ejps.2016.03.020.

9. Mishra, H.; Polak, S.; Jamei, M.; Rostami-Hodjegan, A. Interaction between domperidone and ketoconazole: Toward prediction of consequent QTC prolongation using purely in vitro information. CPT Pharmacometrics Syst. Pharmacol. 2014, 3 (8), e130. DOI: 10.1038/psp.2014.26. 